\title{
Comparison of Thyroid Segmentation Algorithms in Ultrasound and Scintigraphy Images
}

\author{
Jaspreet Kaur, Alka Jindal, \\ PG Student, Department of C.S.E, PEC University of Technology, Chandigarh, \\ Assistant Professor, Department of I.T, PEC University of Technology, Chandigarh.
}

\begin{abstract}
To integrate medical images and image processing, image segmentation plays an essential role. Image segmentation is an operation that separates the image into different segments. There are several image segmentation algorithms that are at present available. In this paper, three segmentation algorithms have been implemented and discussed namely: Active Contour without edges, Localized region Based active contour and Distance Regularized Level Set. To detect the thyroid disorders various imaging modalities are used: MRI, Scintigraphy, SPECT and Ultrasound. Out of these, Ultrasound Imaging and Scintigraphy have been discussed in this paper. The segmentation algorithms have been implemented on these two modalities to segment the thyroid gland.
\end{abstract}

Keywords: Thyroid Gland, Segmentation, Active Contour without edges, Localized region Based active contour and Distance Regularized Level Set.

\section{INTRODUCTION}

The thyroid gland is a butterfly shaped organ. The foremost function of thyroid is to regulate the body's metabolism by making thyroid hormone. There are large numbers of diseases that create abnormalities in thyroid. These abnormalities can be detected through medical images. Thyroid abnormalities can be detected using Ultrasonography, Scintigraphy, SPECT and MRI. Image segmentation is an important tool in Medical Image Processing as it provides the information about an object clinically in terms of its shape and structure.

Segmentation is an important aspect in Medical Imaging as it helps to obtain the region of objects of interest and to detect area, volume etc. there are various imaging modalities used for thyroid such as: MRI, Ultrasound, Scintigraphy and SPECT. Among all the imaging modalities for thyroid, Ultrasonography and Scintigraphy are usually recommended by the physicians. Ultrasound imaging is currently the most diagnostic tool [1]. It is inexpensive and easy to use. Ultrasound is a versatile imaging technique that reveals the internal structure of organs. Ultrasonography is the modality of choice for evaluation and follow-up, because of its sensitivity and convenience. Ultrasound continues to make significant contributions to patient care by reassuring patients and enhancing their quality of life by helping physicians understand their anatomy in ways not possible with other techniques [2].Ultrasound images does not have any hazardous effects on the patient. Thyroid Scan (Scintigraphy) is done in nuclear medical department. Thyroid Scan is performed in order to know the active (working) part of thyroid gland. In this procedure, a radio-iodine medicine is given to the patient and then gamma cameras are used to take pictures of thyroid and show the part of thyroid that uptakes iodine i.e. active or working part of thyroid. Scintigraphy imaging is 2-D imaging that is used to see the working of thyroid [3].
Techniques to process Medical images are continuously being developed. Several methods for segmenting objects from Medical images have been developed. Several methods for segmenting objects from medical images have been developed such as Active contour without edges [3], Variable Background Active Contour (VBAC) [4], Minimization of Region-Scalable Fitting Energy[5], ELM[6], RBF[7]. Among all the Segmentation algorithms, ACWE, Localized Region Based Active Contour [8], and Distance Regularized Level Set (DRLSE) [9] have been discussed in this paper with their application to Thyroid Gland. These segmentation algorithms have been applied on Thyroid Scintigraphy and Ultrasound Images.

The remainder of the paper is organized as follows: Section II discusses the various segmentation algorithms that have implemented on Scintigraphy and Ultrasound Images. Section III presents the results of implementing the algorithms on Scintigraphy images and Ultrasound images. Section IV presents the conclusion.

\section{2. IMAGE}

\section{ALGORITHMS}

SEGMENTATION

An immense number of segmentation algorithms have been developed over the last few years and this number is continuously increasing. In this section, some of the segmentation algorithms that have been implemented on Medical images have been presented such as Active Contour without Edges (ACWE), Localized region Based Active Contour (LRAC) and Distance Regularized Level Set (DRLSE).

\subsection{Active Contour without Edges}

Active Contour without Edges (ACWE) [3] proposed by Chan Vese, is a region based method. It tends to separate the image into two homogeneous regions according to a mean value. Active Contour without Edges is sensitive to initialization. The initial curve can be anywhere in the image, and the interior contours are automatically detected. Active Contour without Edges detects objects whose boundaries are not necessarily defined by gradient or smooth boundaries.

It seeks for minimum energy $\mathrm{E}(\mathrm{u}, \mathrm{v}, \mathrm{C})$ :

$$
\begin{aligned}
\mathrm{E}(\mathrm{u}, \mathrm{v}, \mathrm{C}) & =\mu . \text { Length }(\mathrm{C}) \\
+ & \lambda_{\mathrm{u}} \int_{\text {inside }}^{\cdot}|I(x, y)-u|^{2} \mathrm{dxdy} \\
+ & \lambda_{\mathrm{v}} \int_{\text {outside }}|I(x, y)-v|^{2} \mathrm{dxdy}
\end{aligned}
$$

Where I: $\Omega \rightarrow \mathrm{R}$ is an input image.

$\mathrm{u}, \mathrm{v}$ are the unknown constants representing the average value inside and outside curve. 
$\mu$ and $\lambda_{\mathrm{u}}, \lambda_{\mathrm{v}}>0$ are weights for regularizing terms and fitting term.

The mean value of image inside and outside the curve is defined by:

$u=\frac{\int_{\Omega}^{\cdot} I(x, y) H(\phi(x, y)) d x d y}{\int_{\Omega}^{\cdot}(H(\phi(x, y)) d x d y}$

Active Contour without edge model is based on Mumfordshah Segmentation technique and level set method. This method does not require smoothing the initial image even if the image contains noise. So this method segments well in presence of noise

$$
\boldsymbol{v}=\frac{\int_{\mathbf{\Omega}}^{\cdot} I(x, y)(1-H(\phi(x, y)) d x d y}{\int_{\mathbf{\Omega}}(1-H(\phi(x, y)) d x d y}
$$

Where, $\phi$ : signed distance function and it is reinitialized at each iteration.

\subsection{Localized Region Based Active Contour}

Localized Region based Active Contour [8] is a region based method that uses local region parameters for segmentation segmentation.. In this method, foreground and background are described in terms of smaller local regions. To optimize the local energies, each point is considered separately and moves to minimize the energy computed in its own local region. These local energies are then computed by splitting the local neighborhoods into local interior and local exterior by the evolving curve.

Let ' $I$ ' is a thyroid US image defined in a domain $\Omega$. ' $C$ ' is the evolving curve represented as zero level set of a signed distance function $(\phi)$.

Two spatial variables ' $x$ ' and ' $y$ ' are used in this method to represent a single point in $\Omega$. A characteristic function is used in terms of localized radius parameter ' $r$ ':

$\mathcal{B}(x, y)=\left\{\begin{array}{lc}1, & || x-y \|<r \\ 0, & \text { otherwise }\end{array}\right.$

This function is used to mask local regions. The energy function in terms of force function is defined as:

$$
E(\phi)=\int_{\Omega x}^{\cdot} \delta \phi(x) \int_{\Omega y} \mathcal{B}(x, y) F(I(y), \phi(y)) d y d x
$$

The localized mean intensities of interior and exterior region of contour localized by $\mathcal{B}(x, y)$ at a point $x$ are denoted by ' $u_{x}$ ' and ' $v_{x}$ ' are defined as:

$$
\begin{aligned}
& u_{\mathrm{x}}=\frac{\int_{\Omega y} \mathcal{B}(x, y) \cdot \mathcal{H} \phi(y) \cdot I(y) d y}{\int_{\Omega y}^{\cdot} \mathcal{B}(x, y) \cdot \mathcal{H} \phi(y) d y} \\
& v_{\mathrm{x}}=\frac{\int_{\Omega y} \mathcal{B}(x, y) \cdot(1-\mathcal{H} \phi(y)) \cdot I(y) d y}{\int_{\Omega y}^{\cdot} \mathcal{B}(x, y) \cdot(1-\mathcal{H} \phi(y)) d y}
\end{aligned}
$$

These localized parameters determine local energies at each point along the curve.

After selecting one of the localized energy measures, it is multiplied with Dirac function $\delta \phi(x)$ in order to capture broader range of objects. For every point $x$ selected by Dirac function $\delta \phi(x)$ a localized mask $\mathcal{B}(x, y)$ is applied to it to ensure that $\mathrm{F}$ operates only in local region about $x$. The final energy is defined as:

$$
\begin{gathered}
E(\phi)=\int_{\Omega x}^{\cdot} \delta \phi(x) \int_{\Omega y}^{\cdot} \mathcal{B}(x, y) \cdot F(I(y), \phi(y)) d y d x+ \\
\lambda \int_{\Omega x}^{\cdot} \delta \phi(x)\|\nabla \phi(x)\| d x
\end{gathered}
$$

The convergence time for segmentation decreases as radius size increases.

\subsection{Distance Regularized Level Set}

Chunming $\mathrm{Li}$, Chenyang $\mathrm{Xu}$, et al. aimed to design a segmentation method that is edge based and yields less numerical errors [9]. The authors proposed a new variational level set formulation in which the regularity of the level set function is intrinsically maintained during the level set evolution. The level set evolution is derived as the gradient flow that minimizes energy functional with a distance regularization term and an external energy that drives the motion of the zero level set toward desired locations.It also reduces the number of iterations required for segmentation. The algorithm first filters the image using a Gaussian kernel filter and then calculates the energy function as:

$\mathrm{E}(\phi)=\mu R_{\mathrm{p}}(\phi)+\lambda \cdot \mathcal{L}_{\mathrm{g}}(\phi)+\alpha A_{\mathrm{g}}(\phi)$

Where, $R_{\mathrm{p}}$ is level ser regularizing term;

$R_{\mathrm{p}}(\phi) \triangleq \int_{\Omega} p(|\nabla \phi|) d x$

$\mathrm{P}$ is the potential energy. $\mathcal{L}_{\mathrm{g}}$ and $A_{\mathrm{g}}$ are the energy functions. This method allows more flexible and efficient initialization than generating a signed distance function as the initial LSF The distance regularization term is defined with a potential function such that the derived level set evolution has a unique forward-and-backward (FAB) diffusion effect, which is able to maintain a desired shape of the level set function, particularly a signed distance profile near the zero level set.

\section{IMPLEMENTATION DETAILS}

In this section, the results of implementing the above discussed algorithm have been presented and compared. The algorithms were first implemented on Scintigraphy Images and then on ultrasound Images. The presented algorithms were implemented in MATLAB R2008b version.

\subsection{Scintigraphy Images}

The Scintigraphy images were collected from various web sources. Three algorithms namely Active Contour without edges; Localized region Based active contour and Distance Regularized Level Set were implemented on Scintigraphy images. The algorithms were compared in terms of time and no of iterations taken by them to segment the image. The results of scintigraphy images are shown in fig. 1. Fig 1(a) shows the thyroid scintigraphy original image (b) shows the segmented image after applying ACWE (c) shows the image after applying LRAC and (d) shows the segmented image after applying DRLSE. It is evident from the images that the results of Localized region based Active contour are better than the other algorithms. A comparison of these segmentation algorithms have been shown in fig. 3 in terms of time and no of iterations. Following table shows the corresponding values for the graph. 
Table 1. Graph values

\begin{tabular}{|l|l|l|l|}
\hline \multirow{2}{*}{} & \multicolumn{2}{|l|}{ Scintigraphy Images } \\
\cline { 2 - 4 } & ACWE & LRAC & DRLSE \\
\hline $\begin{array}{l}\text { No. of } \\
\text { iterations }\end{array}$ & 270 & 160 & 410 \\
\hline Time(Sec) & 3.8 & 2.9 & 45 \\
\hline
\end{tabular}

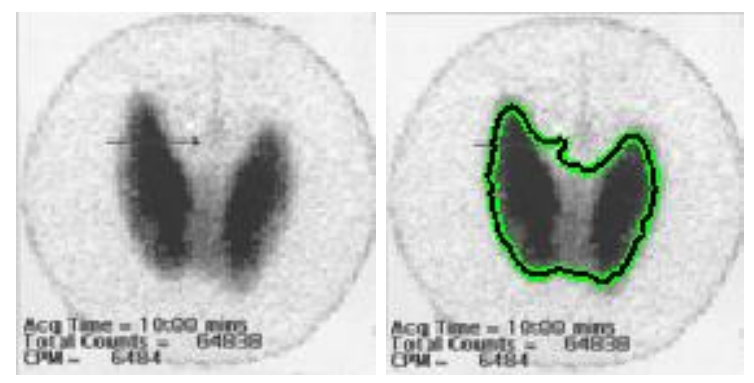

Fig 1 (a) Thyroid Scintigraphy original image (b) results after ACWE (c) LRAC
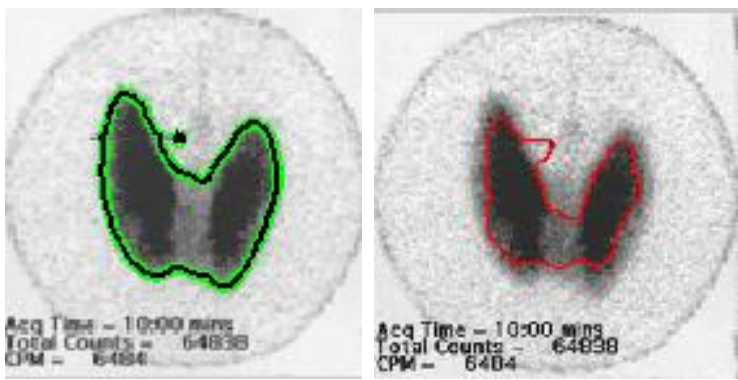

(d) DRLSE
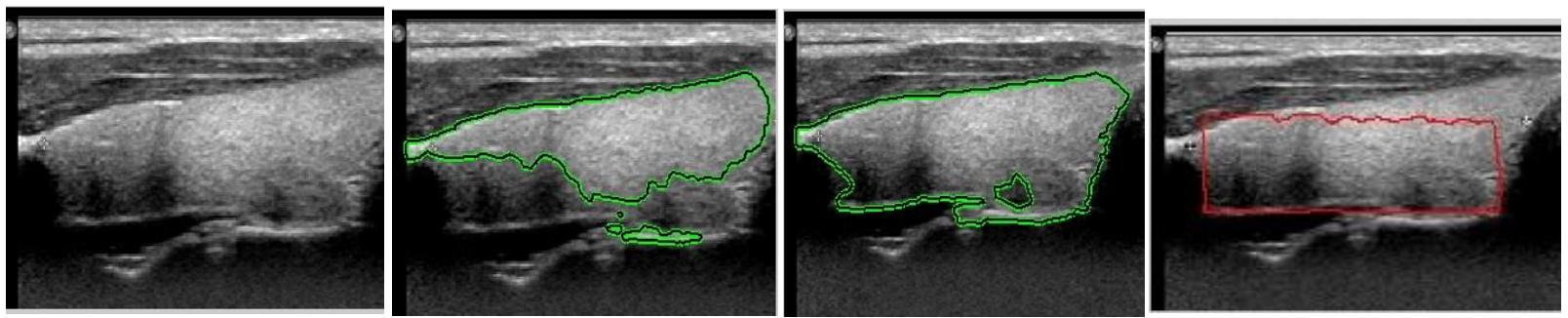

Fig 2 (a) Thyroid US original image (b) results after ACWE (c) LRAC (d) DRLSE

Fig 2 (a) shows the thyroid US original image (b) shows the segmented image after applying ACWE and (c) shows the segmented result after applying LRAC and (d) shows the segmented result after DRLSE. Localized region based active contour method segments the image better than the ACWE and DRLSE. Comparison has been made on the basis of Precision [10] factor which is shown in fig 4. Precision is calculated as:

$\mathrm{P}=\frac{\text { Total number of images Correctly Segmented }}{\text { Total number of Query Images }} * 100 \%$

The algorithms were implemented on a dataset of 10 Scintigraphy images. Precision values were calculated for these 10 Scintigraphy images. Precision percentage for ACWE is $57 \%$ and for LRAC is $80 \%$.

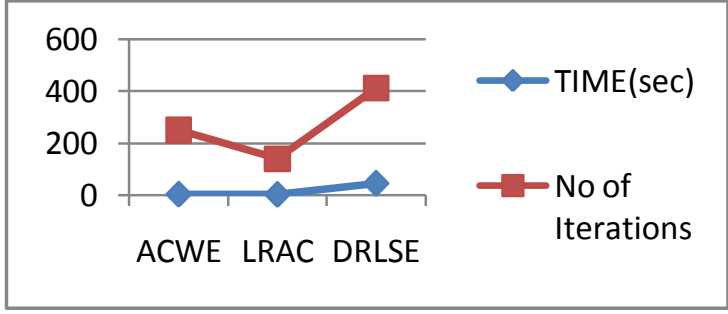

Fig 2: Comparison of segmentation algorithms in Scintigraphy Images.

The following graph shows the value for precision :

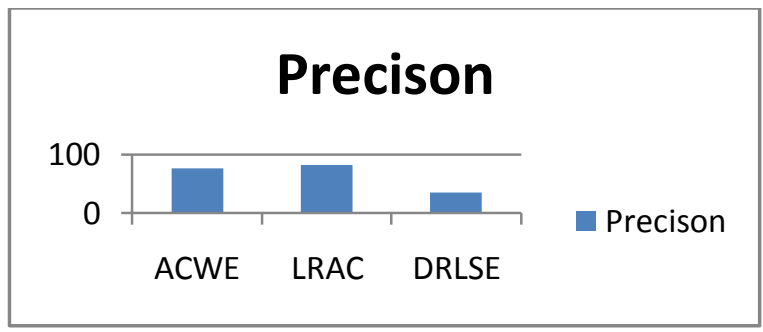

Fig. 4 Comparison of Algorithms based on Precision 


\section{CONCLUSION}

To inspect the abnormalities in thyroid gland various imaging modalities are used. In this paper, two thyroid imaging modalities were discussed: Ultrasound Imaging and Scintigraphy. Segmentation algorithms were implemented on both the imaging modalities. Furthermore, the segmentation algorithms were compared based on several parameters in both the imaging modalities. The best image segmentation algorithm was Localized Region Based as it took less time and less number of iterations in Scintigraphy images. This algorithm had a precision of $80 \%$ in segmenting the US images.

In future work, comparison of more segmentation algorithms could be implemented on thyroid images.

\section{REFERENCES}

[1]. Chuan-Yu Chang, Senior Member, IEEE, Yue-Fong Lei, Chin-Hsiao Tseng, and Shyang-Rong Shih, "Thyroid Segmentation and Volume Estimation in Ultrasound Images", IEEE TRANSACTIONS ON BIOMEDICAL ENGINEERING, VOL. 57, NO. 6, JUNE 2010.

[2]. D.Selvathi , V.S.Sharnitha, "Thyroid Classification and Segmentation in Ultrasound Images Using Machine Learning Algorithms," Proceedings of 2011 International Conference on Signal Processing, Communication, Computing and Networking Technologies (ICSCCN 2011).

[3]. T.F. Chan, L.A. Vessse,"Active Contours edges”, IEEE tras. Image Processing, Feb. 2001, pp. 266-277.

[4].Dimitris K. Iakovidis - Michalis A. Savelonas ,Stavros A. Karkanis and Dimitris E. Maroulis, " a genetically optimized level set approach to segmentation of thyroid ultrasound images," Published online: 16 June 2007 Springer Science+Business Media, LLC 2.

[5]. Chunming Li, Chiu-Yen Kao, John C. Gore, and Zhaohua Ding, "Minimization of Region Scalable Fitting Energy for Image Segmentation," IEEE TRANSACTIONS ON IMAGE PROCESSING, VOL. 17, NO. 10, OCTOBER 2008.

[6] D.Selvathi, V.S.Sharnitha, "Thyroid Classification and Segmentation in Ultrasound Images Using Machine Learning Algorithms," Proceedings of 2011 International Conference on Signal Processing, Communication, Computing and Networking Technologies (ICSCCN 2011) [7].

Chuan-Yu Chang and Chin-Hsiao Tseng et al "Thyroid Segmentation and Volume Estimation in Ultrasound Images" IEEE TRANSACTIONS ON BIOMEDICAL ENGINEERING, VOL. 57, NO. 6, JUNE 2010.

[8]Shawn Lankton, Allen Tannenbaum,"Localizing RegionBased Active Contours", IEEE Transactions on Image Processing, Vol. 17, No. 11, November 2008.

[9]. Chunming

$\mathrm{Li}$, Chenyang $\mathrm{Xu}$, Changfeng Gui, Martin D. Fox,"Distance Regularized Level Set Evolution and Its Application to Image Segmentation", IEEE Transactions on Image Processing, Vol. 19, No. 12, December 2010.

[10]. Peter LUKAC, Robert HUDEC, Miroslav BENCO,et al. "Simple Comparison of Image Segmentation", 978-161284-324-7/11 2011 IEEE 